\title{
Galectin-3-induced oxidized low-density lipoprotein promotes the phenotypic transformation of vascular smooth muscle cells
}

\author{
LEI TIAN, KAN CHEN, JIATIAN CAO, ZHIHUA HAN, LIN GAO, YUE WANG, \\ YUQI FAN and CHANGQIAN WANG \\ Department of Cardiology, Shanghai Ninth People's Hospital, School of Medicine, \\ Shanghai Jiaotong University, Shanghai 200011, P.R. China
}

Received September 28, 2014; Accepted June 25, 2015

DOI: $10.3892 / \mathrm{mmr} .2015 .4075$

\begin{abstract}
Oxidized low-density lipoprotein (oxLDL) is involved in the pathological phenotypic transformation of vascular smooth muscle cells in atherosclerosis. Galectin-3 also has an important role in atherosclerosis. However, little is currently known regarding the effects of galectin-3 on the oxLDL-induced phenotypic transformation of vascular smooth muscle cells. In the present study, primary culture human umbilical vascular smooth muscle cells were treated with various oxLDL concentrations $(0-50 \mu \mathrm{g} / \mathrm{ml})$ for $72 \mathrm{~h}$, and phenotypic changes were subsequently recorded. The results of the present study suggested that oxLDL increases the expression levels of galectin-3, and induces the phenotypic transformation of vascular smooth muscle cells. The oxLDL-induced cells exhibited increased expression levels of osteopontin, a smooth muscle synthetic protein, and calponin and $\alpha$-actin, smooth muscle contractile proteins. The oxLDL-induced changes in cellular phenotype were associated with increased migration, proliferation, and phagocytosis. Concordant with these results, oxLDL-treated smooth muscle cells exhibited activation of canonical Wnt signaling, as determined by an increase in the protein expression levels of $\beta$-catenin. Silencing of galectin-3 by small interfering RNA reversed the phenotypic transformation and functional changes observed in the oxLDL-treated cells, suggesting these changes were dependent on the activation of galectin-3. In addition, galectin-3 knockdown decreased the protein expression levels of $\beta$-catenin in both the cytoplasm and nucleus; however, the mRNA expression levels of $\beta$-catenin remained unchanged. These results suggest that galectin-3 is responsible for the phenotypic transformation
\end{abstract}

Correspondence to: Dr Yuqi Fan or Dr Changqian Wang, Department of Cardiology, Shanghai Ninth People's Hospital, School of Medicine, Shanghai Jiaotong University, 639 Zhizaoju Road, Shanghai 200011, P.R. China

E-mail: fanyuqi2014@126.com

E-mail: jiuyuanwcq@163.com

Key words: oxidized low-density lipoprotein, galectin-3, vascular smooth muscle cell, phenotypic transformation, Wnt/ $\beta$-catenin signaling of human umbilical vascular smooth muscle cells, and the canonical Wnt $/ \beta$-catenin signaling pathway may be involved in this process.

\section{Introduction}

Atherosclerosis is a pathological process leading to numerous fatal diseases, such as coronary heart disease, cerebral infarction, and cerebral hemorrhage. The phenotypic change of vascular smooth muscle cells (VSMCs) from a differentiated state to a dedifferentiated state is critical to the progression of atherosclerosis. Numerous studies have been carried out $(1,2)$; however, the molecular mechanisms underlying the phenotypic transformation of VSMCs remain to be elucidated.

Galectin-3 (gal-3), which is a $29-35 \mathrm{kDa}$ protein, is a member of the $\beta$-galactoside-binding lectin family (3). Gal-3 is composed of a C-terminal carbohydrate-recognition domain, a collagen-like internal R-domain, and an N-terminal domain that promotes lectin oligomerization $(3,4)$. Gal-3 is present in the cytoplasm, nucleus, extracellular space, and is also bound to the cell surface (3). Gal-3 can be secreted into the extracellular matrix and serum, where it binds to protein laminin, fibronectin, and collagen IV. Although gal-3 is present in almost every organ of the body, its contents vary greatly. A recent study demonstrated that gal-3 may serve as a diagnostic and prognostic marker for cardiovascular diseases (5). A previous study demonstrated that gal-3 has an important pathophysiological role in cardiovascular remodeling by promoting changes including cardiac hypertrophy, fibrosis, arterial stiffness, as well as inflammation and oxidative stress (6). Gal-3 may also serve as a tool to predict future heart failure, and is associated with heart failure morbidity and mortality (7). In addition, gal-3 has been shown to induce angiogenesis and promote new blood vessel formation (8).

Recent studies have reported that gal-3 has a role in atherogenesis. An increase in the expression levels of gal-3 is associated with the developmental process of atherogenesis (9). In addition, gal-3 is necessary for the inflammatory response induced by aldosterone in VSMCs (6). Activation and dedifferentiation of VSMCs are the principal pathological changes that occur within the blood vessel wall during atherogenesis. Activation of VSMCs also contributes 
to plaque instability (1). However, the mechanism underlying this pathological process remains to be elucidated.

Oxidized low-density lipoprotein (oxLDL) has been widely reported to have a role in atherogenesis (10). In addition, oxLDL increases the ability of macrophages to uptake lipid products (11), and promotes the migration and proliferation of VSMCs (12). Considering the importance of oxLDL in inducing atheroma, the present study aimed to investigate whether gal-3 may function together with oxLDL in atherogenesis. To demonstrate this hypothesis, the role of gal-3 in the oxLDL-induced phenotypic and functional changes of VSMCs was examined. The molecular mechanism underlying this process was also investigated.

\section{Materials and methods}

Reagents. Dulbecco's modified Eagle's medium (DMEM), fetal bovine serum (FBS), and penicillin/streptomycin $(10,000 \mathrm{U} / \mathrm{ml}$ each) were purchased from Gibco Life Technologies (Carlsbad, CA, USA). TRIzol ${ }^{\circledR}$ reagent for RNA isolation was purchased from Invitrogen Life Technologies (Carlsbad, CA, USA). A Reverse Transcriptase kit (cat. no. RR037A) and SYBR Premix Ex Taq II (cat.no. RR820A) were purchased from Takara Biotechnology Co., Ltd. (Dalian, China). A Cell Counting kit-8 (CCK-8) assay was purchased from Dojindo Molecular Technologies, Inc. (Kumamoto, Japan). Oil Red O was purchased from Sigma-Aldrich (St. Louis, MO, USA). OxLDL was purchased from Peking Union-Biology Co., Ltd. (Beijing, China). The primary antibodies targeting smooth muscle $\alpha$-actin (SMA; cat. no. sc-53142), gal-3 (cat. no. sc-20157), and GAPDH (cat. no. sc-48166) were purchased from Santa Cruz Biotechnology, Inc. (Dallas, TX, USA). The anti- $\beta$-catenin antibody (cat. no. 8480) was purchased from Cell Signaling Technology, Inc. (Danvers, MA, USA). The monoclonal antibody targeting osteopontin (OPN; cat. no. ab91655) was purchased from Abcam (Cambridge, UK). The goat anti-rabbit secondary antibody (cat. no. A-21109) and the goat anti-mouse secondary antibody (cat. no. A-21058) were purchased from Invitrogen Life Technologies.

Cells culture. A primary culture of human umbilical smooth muscle cells (HUSMCs) was established as previously described (13). Briefly, HUSMCs were collected by explant outgrowth of a segment of human umbilical cord obtained during a cesarean section procedure. Endothelial cells were removed by scraping the luminal surface of the vessel with a cotton swab, and the adventitia was mechanically stripped away. Primary cultures were maintained in DMEM supplemented with $20 \%$ FBS and $1 \%$ penicillin/streptomycin. The cells obtained between the 4th and 10th passage were used for further experimentation. The experimental procedures of the present study complied with the principles of the Declaration of Helsinki, and were approved by the Ethics Committee of the Shanghai Ninth People's Hospital (Shanghai, China). Written informed consent was obtained from all patients.

Small interfering (si)RNA transfection. Gal-3 expression was inhibited by transfection with a siRNA specific to gal-3. Gal-3 siRNA was transiently transfected into the cells using Lipofectamine ${ }^{\circledR} 2000$ (Invitrogen Life
Technologies), according to the manufacturer's instructions. Briefly, 5x10 $10^{5}$ HSMCs per well were cultured in 6-well plates to $75 \%$ confluence. The cells were then transfected with 100 pmol siRNA duplexes using $5 \mu$ l Lipofectamine ${ }^{\circledR}$ 2000 and DMEM. Following a $72 \mathrm{~h}$ incubation at $37^{\circ} \mathrm{C}$ the cells were harvested for analysis. The human gal-3 siRNA sequence was 5'-CCUCGCAUGCUGAUAACAATT-3', and the scrambled siRNA sequence was 5'-UUGUUAUCA GCAUGCGAGGTT-3'. siRNA were synthesized by Biotend (Shanghai, China).

Cell proliferation assay. Cell proliferation was measured using the CCK-8 assay. Following cellular transfection with gal-3 siRNA or scramble siRNA for $24 \mathrm{~h}$, cells were plated into 96-well plates, at a density of $5 \times 10^{3}$ cell/well. The HUSMCs were subsequently incubated for 24 or $48 \mathrm{~h}$ in the absence or presence of $50 \mu \mathrm{g} / \mathrm{ml}$ oxLDL, and cell proliferation was measured using the CCK-8 assay according to the manufacturer's instructions, at 0,24 or $48 \mathrm{~h}$.

Migration assay. The migration assay was performed as previously described (14). Briefly, following cellular transfection with gal-3 siRNA or scramble siRNA, the HUSMCs were resuspended in $200 \mu \mathrm{l}$ serum-free DMEM, and $5 \times 10^{4}$ HUSMCs were loaded into the upper chambers of a transwell chamber (Corning Inc., Corning, NY, USA). The lower chambers were filled with $400 \mu \mathrm{l}$ DMEM in the presence or absence of $10 \mu \mathrm{g} / \mathrm{ml}$ oxLDL. The cells were then incubated at $37^{\circ} \mathrm{C}$ for $24 \mathrm{~h}$. The lower side of the filter was washed three times with phosphate-buffered saline prior to being fixed with $4 \%$ paraformaldehyde for $20 \mathrm{~min}$. The nuclei were stained with 4',6-diamino-2-phenylindole (DAPI; 1:1,000; Sigma-Aldrich) for $5 \mathrm{~min}$ at room temperature. The cells were counted using an Eclipse TS100 microscope (Nikon Corporation, Tokyo, Japan) in three random high-power fields (magnification, x100) for each well.

Immunofluorescence staining and confocal laser microscopy. The cells $\left(5 \times 10^{5}\right)$ were seeded onto flame-sterilized coverslips and placed into 6-well tissue culture plates. The cells were then transfected with gal-3 siRNA or scramble siRNA for $24 \mathrm{~h}$. The cells were subsequently fixed with $4 \%$ paraformaldehyde for $15 \mathrm{~min}$, permeabilized with $0.1 \%$ Triton X-100 (Sangon Biotech Co., Ltd., Shanghai, China) for $20 \mathrm{~min}$, blocked with $1 \%$ bovine serum albumin (Sigma-Aldrich) for $1 \mathrm{~h}$, and incubated with specific primary antibody overnight at $4^{\circ} \mathrm{C}$. The cells were incubated with Alexa Fluor 488-conjugated goat anti-rabbit immunoglobulin G (Invitrogen Life Technologies) for $1 \mathrm{~h}$ at room temperature. The nuclei were stained with DAPI $(1: 1,000)$ for $5 \mathrm{~min}$ at room temperature. The protein expression levels of $\beta$-catenin were then quantified using a FluoView $^{\mathrm{TM}}$ FV1000 confocal laser scanning microscope (Olympus Corporation, Tokyo, Japan).

Oil Red $O$ staining assay. The Oil Red $\mathrm{O}$ staining assay was performed as previously described (15). Briefly, HUSMCs $\left(5 \times 10^{5}\right)$ were seeded on sterile coverslips in 6-well plates. The cells were then transfected with gal-3 siRNA or scramble siRNA for $24 \mathrm{~h}$, prior to being treated with $50 \mu \mathrm{g} / \mathrm{ml}$ oxLDL for $48 \mathrm{~h}$. The cells were washed three times with PBS, fixed 
in $4 \%$ paraformaldehyde for $20 \mathrm{~min}$ and then stained with $0.5 \%$ Oil Red $\mathrm{O}$ for $20 \mathrm{~min}$ in order to identify lipid droplets in the cytoplasm using an Eclipse TS100 microscope at 40x magnification.

Reverse transcription-quantitative polymerase chain reaction (RT-qPCR) analysis. Total RNA was extracted using TRIzol ${ }^{\circledR}$ reagent, according to the manufacturer's instructions. Total RNA was reverse-transcribed into cDNA using a Reverse Transcriptase kit (cat. no. RR037A), and RT-qPCR was performed using SYBR Premix Ex Taq II (cat. no. RR820A), with gene-specific primers, on an Applied Biosystems 7500 Real-Time PCR system (Applied Biosystems Life Technologies, Foster City, CA, USA), according to the manufacturer's instructions. The primers targeting human gal-3, $\beta$-catenin, calponin, SMA, and OPN were as follows: Gal-3, forward 5'-GGCCACTGATTGTGCCTTAT-3', and reverse 5'-TGCAACCTTGAAGTGGTCAG-3'; $\beta$-catenin, forward 5'-GCCGGCTATTGTAGAAGCTG-3', and reverse 5'-GAGTCCCAAGGAGACCTTCC-3'; Calponin, forward 5'-ATGTGAGGAGGGAAGAGTGTG-3', and reverse 5'-CGGTTGAAGTGAGCAGAGG-3'; SMA, forward 5'-AGCGTGGCTACTCCTTCGTGAC-3', and reverse 5'-GCTCGTTGCCGATGGTGATGAC-3'; OPN, forward 5'-TGAGTCTGGAAATAACTAATGTGTTTGA-3', and reverse 5'-GAACATAGACATAACCCTGAAGCTTTT-3'; and GAPDH, forward 5'-TGATGACATCAAGAAGG TGGTGAAG-3', and reverse 5'-TCCTTGGAGGCCA TGTGGGCCAT-3'. The primers were synthesized by Sangon Biotech Co., Ltd. The PCR cycling conditions were as follows: Initial denaturation at $95^{\circ} \mathrm{C}$ for $30 \mathrm{sec}$, followed by 40 cycles at $95^{\circ} \mathrm{C}$ for $5 \mathrm{sec}, 60^{\circ} \mathrm{C}$ for $34 \mathrm{sec}$ and $95^{\circ} \mathrm{C}$ for $15 \mathrm{sec}$, and finally $60^{\circ} \mathrm{C}$ for $1 \mathrm{~min}$ and $95^{\circ} \mathrm{C}$ for $15 \mathrm{sec}$. The relative mRNA expression levels were calculated using the comparative cycle threshold (CT) method $\left(2^{-\Delta \Delta C T}\right)(16)$.

Western blot analysis. The cells were lysed using a lysis buffer containing $150 \mathrm{mM} \mathrm{NaCl}, 10 \mathrm{mM}$ Tris ( $\mathrm{pH} 7.5), 5 \mathrm{mM}$ EDTA, 1\% Triton X-100, 1 mM phenylmethylsulfonyl fluoride, $10 \mathrm{mg} / \mathrm{ml}$ leupeptin, $10 \mathrm{mg} / \mathrm{ml}$ pepstatin, and $10 \mathrm{mg} / \mathrm{ml}$ aprotinin for $30 \mathrm{~min}$ on ice. Protein concentrations were measured using a Bicinchoninic Acid Protein Assay kit (Pierce Biotechnology, Inc., Rockford, IL, USA). The lysates $(20 \mu \mathrm{g})$ were separated by $10 \%$ SDS-PAGE, and transferred onto nitrocellulose membranes (EMD Millipore, Billerica, MA, USA). The membranes were then blocked with 5\% non-fat dry milk in Tris-buffered saline and Tween 20 (TBST; $100 \mathrm{mM} \mathrm{NaCl,} 10$ mM Tris-HCl, pH 7.4, and $0.1 \%$ Tween 20 ) for $1 \mathrm{~h}$ at room temperature. The blots were incubated with the various primary antibodies at a dilution of 1:1,000 in TBST, overnight at $4^{\circ} \mathrm{C}$, and then washed twice with TBST buffer at room temperature, prior to incubation for $1 \mathrm{~h}$ with the appropriate peroxidase-conjugated secondary antibodies (1:5,000). All signals were detected using Odyssey ${ }^{\circledR}$ Clx Infrared Imaging system (LI-COR Biosciences, Lincoln, NE, USA). Protein quantification was achieved by measuring the band intensity using Quantity One ${ }^{\circledR}$ 4.6.2 software (Bio-Rad Laboratories, Inc., Hercules, CA, USA).

Statistical analysis. All data are expressed as the mean \pm standard deviation. Statistical analyses were performed using
SPSS 13.0 software (SPSS, Inc., Chicago, IL, USA). The data were analyzed using a one-way analysis of variance (ANOVA) and Student-Newman-Keuls post-hoc test. All of the data did not pass the normality test, therefore, a non-parametric ANOVA (Kruskal-Wallis test) was used, as appropriate. $\mathrm{P}<0.05$ was considered to indicate a statistically significant difference. All experiments were performed in triplicate.

\section{Results}

OxLDL induces the phenotypic transformation of HUSMCs, and increases the expression levels of gal-3. According to a previous study (17), minimally-oxidized LDL may induce phenotypic transformation of coronary artery smooth muscle cells. The present study demonstrated that oxLDL directly induced significant changes in smooth muscle cell phenotype, following cellular treatment with various oxLDL concentrations $(0-50 \mu \mathrm{g} / \mathrm{ml})$. Treatment with oxLDL induced a marked increase in the expression levels of smooth muscle synthetic related protein OPN, and contractile related proteins calponin and SMA. OxLDL also increased the protein expression levels of gal-3 (Fig. 1A and B).

Silencing gal-3 reverses the oxLDL-induced phenotypic transformation of HUSMCs. To investigate the role of gal-3 in the oxLDL-induced phenotypic changes of HUSMCs, gal-3 knockdown was performed. Following transfection with siRNA, the expression of endogenous gal-3 was inhibited, and the phenotypic changes of HUSMCs were assessed. Gal-3-specific siRNA reduced the mRNA and protein expression levels of gal-3 by 95 and $45 \%$, respectively. The non-targeting siRNA had no effect on gal-3 expression. Neither gal-3 nor the non-targeting siRNA induced a non-specific knockdown of GAPDH. Furthermore, gal-3 knockdown significantly inhibited both the oxLDL-induced mRNA and protein expression levels of OPN, calponin, and SMA (Fig. 2A-C). These results suggest that gal-3 may be involved in the oxLDL-induced phenotypic transformation of HUSMCs.

Silencing gal-3 reduces the oxLDL-induced activation of HUSMCs. Previous studies (18-20) have reported that oxLDL may promote the activation of VSMCs, including migration, proliferation, and phagocytosis. The present study investigated whether gal-3 was involved in the oxLDL-induced activation of HUSMCs. Migration assays were performed in order to determine the effects of gal-3 silencing on cell motility, and cell numbers, which were determined using a microscope. Knockdown of endogenous gal-3 markedly reduced oxLDL-mediated cell migration in vitro (Fig. $3 \mathrm{~A}$ and $\mathrm{B}$ ). A previous study reported that $50 \mu \mathrm{g} / \mathrm{ml}$ oxLDL induced the proliferation of HUSMCs at $24 \mathrm{~h}$ (21). The present study demonstrated that silencing gal-3 reduced cell proliferation, and $50 \mu \mathrm{g} / \mathrm{ml}$ oxLDL induced proliferation at $24 \mathrm{~h}$ and $48 \mathrm{~h}$ (Fig. 3C). These results suggest that gal-3 may affect the proliferation of HUSMCs. The effects of gal-3 knockdown on cell phagocytosis were also investigated. The cells were treated with oxLDL for $48 \mathrm{~h}$, following which lipid accumulation was stained with Oil Red O. A marked increase in Oil Red $\mathrm{O}$ staining in the control cells incubated with oxLDL was observed, as compared with the cells transfected with 
A

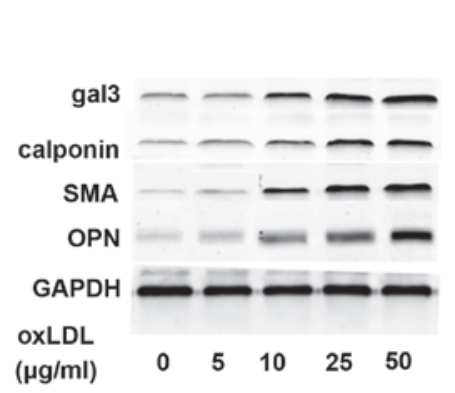

B

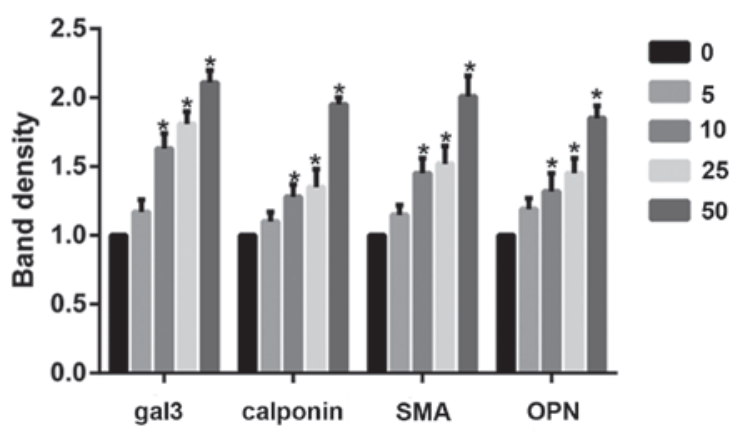

Figure 1. (A) Oxidized low-density lipoprotein (oxLDL) induces phenotypic changes in human umbilical smooth muscle cells (HUSMCs), and increases the expression levels of galectin-3 (gal-3). The cells were treated with various concentrations of oxLDL $(0-50 \mu \mathrm{g} / \mathrm{ml})$ for $48 \mathrm{~h}$, and the expression levels of gal-3, calponin, smooth muscle $\alpha$-actin (SMA), and osteopontin (OPN) were measured by western blotting. (B) The protein expression levels of GAPDH were used as a control. The respective densitometric measurement results are given, and the band intensities of the HUSMCs were defined by the control and set to 1. Data are presented as the mean \pm standard deviation. ${ }^{*} \mathrm{P}<0.05$, vs. the control.

A

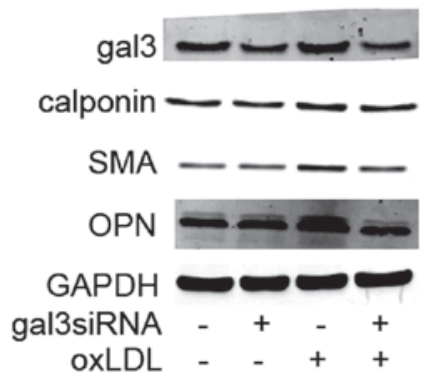

B

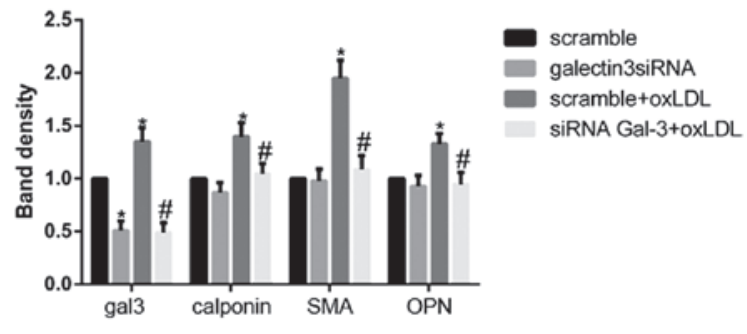

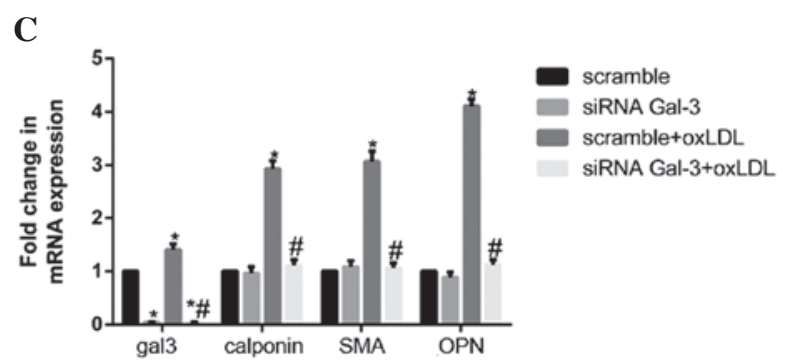

Figure 2. Silencing of galectin-3 (gal-3) reversed the oxidized low-density lipoprotein (oxLDL)-induced phenotypic transformation of human umbilical smooth muscle cells (HUSMCs). HUSMCs were transfected with gal-3-specific small interfering (si)RNA for $24 \mathrm{~h}$, and then cultured with $50 \mu \mathrm{g} / \mathrm{ml}$ oxLDL for $48 \mathrm{~h}$. The mRNA and protein expression levels of gal-3, smooth muscle $\alpha$-actin (SMA), calponin, and osteopontin (OPN) were measured by reverse transcription-quantitative polymerase chain reaction (RT-qPCR) and western blotting. (A and B) Western blotting and (C) RT-qPCR results of gal-3, calponin, OPN and SMA are shown. (B) The respective densitometric measurement results are given. The protein expression levels of gal-3, calponin, OPN and SMA were normalized to those of GAPDH. Band density of HUSMCs transfected with scramble siRNA was defined as control and set to 1 . Data are presented as the mean \pm standard deviation. ${ }^{*} \mathrm{P}<0.05$, vs. the control. ${ }^{~} \mathrm{P}<0.05$, vs. oxLDL.

gal-3 siRNA (Fig. 3D). These results suggest that gal-3 has an important role in the oxLDL-induced activation of HUSMC.

Gal-3 silencing inhibits the expression of $\beta$-catenin. To further elucidate the molecular mechanism underlying the gal-3-mediated oxLDL-induced activation and transformation of HUSMCs, the present study investigated whether Wnt/ $\beta$-catenin signaling was involved in the process. The basis for these experiments was provided by the well-documented role of gal-3 in Wnt/ $\beta$-catenin signaling (22), as well as the association between Wnt/ $\beta$-catenin signaling and phenotypic changes in murine VSMCs (23). Following treatment with oxLDL, the protein expression levels of $\beta$-catenin increased; however, the mRNA expression levels of $\beta$-catenin remained unchanged. The present study subsequently investigated the expression levels of $\beta$-catenin using RT-qPCR and western blotting, following gal-3 knockdown. Silencing of gal-3 significantly reduced the oxLDL-induced protein expression levels of $\beta$-catenin (Fig. 4A and B). However, no changes were observed in the mRNA expression levels of $\beta$-catenin following treatment with gal-3 siRNA (Fig. 4C). In the gal3 siRNA-transfected and control cells, the subcellular distribution of $\beta$-catenin was examined by immunofluorescence staining and confocal microscopy. Reduced expression levels of $\beta$-catenin were observed not only in the cytoplasm but also in the nucleus (Fig. 4D). These data suggest that gal-3 has 
A
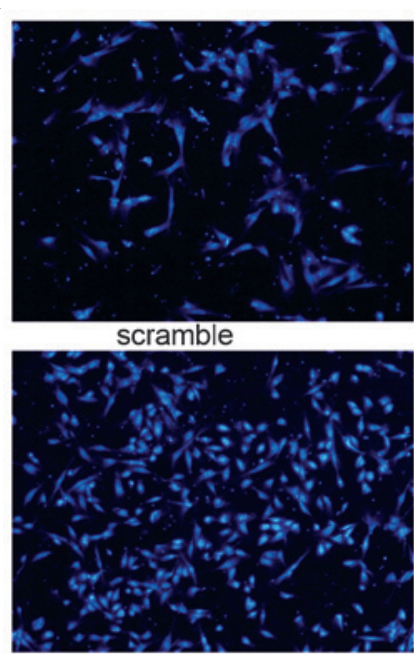

scramble+oxLDL

D

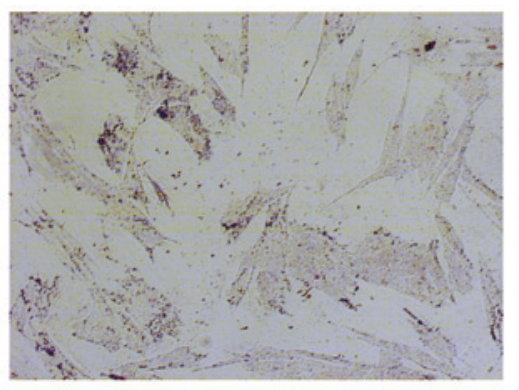

oxLDL
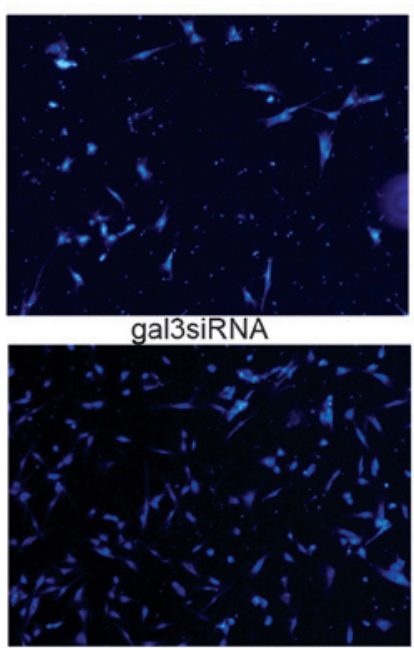

gal3siRNA+oxLDL
B

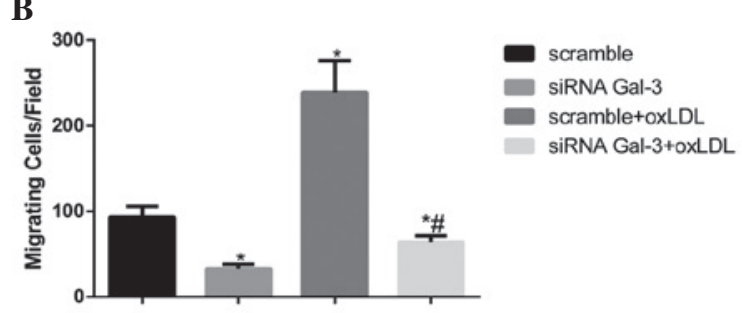

$\begin{array}{cc}\text { C } & 4 \\ & 3 \\ & \\ & \\ & \\ & \\ & \end{array}$

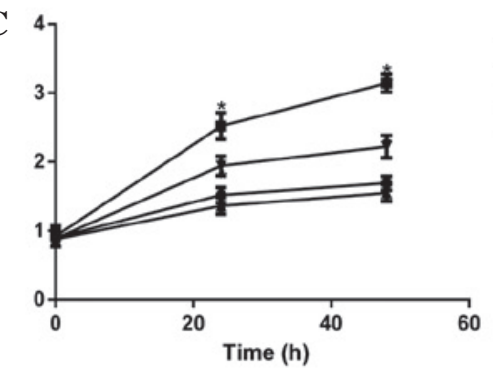

* scramble

- oxLDL+scramble

$\bullet$ oxLDL+gal3siRNA

Figure 3. Galectin-3 (gal-3) knockdown reduces the oxidized low-density lipoprotein (oxLDL)-induced activation of human umbilical smooth muscle cells (HUSMCs). (A) Following transfection with either control or gal-3-specific small interfering (si)RNA for 48 h, cellular migration was analyzed using a transwell assay. The cells that migrated from the upper to the lower chamber were counted in five non-overlapping fields under a microscope (magnification, $\mathrm{x} 100$ ). The cells that migrated to the lower surface of each chamber are shown. (B) The cells transfected with scramble siRNA were defined as control and set to 100 . Following transfection with either control or gal-3-siRNA for $24 \mathrm{~h}$, HUSMCs were incubated for $48 \mathrm{~h}$ in the absence or presence of $50 \mu \mathrm{g} / \mathrm{ml}$ oxidized low-density lipopolyprotein (oxLDL). "P<0.05, vs. control; ${ }^{*} \mathrm{P}<0.05$, vs. oxLDL. (C) Following transfection, the HUSMCs were incubated for 24 or $48 \mathrm{~h}$ in the absence or presence of $50 \mu \mathrm{g} / \mathrm{ml}$ oxLDL. A Cell Counting kit- 8 assay was used to detect cell proliferation. The cells transfected with scramble siRNA were defined as control. (D) The HUSMCs were stained with Oil Red O in order to detect neutral lipids. Representative images (magnification, x40) of the cells stained with Oil Red O are shown. Data are presented as the mean \pm standard deviation. ${ }^{*} \mathrm{P}<0.05$, vs. the control. ${ }^{*} \mathrm{P}<0.05$, vs. oxLDL.

an important role in oxLDL-induced increases in $\beta$-catenin expression.

\section{Discussion}

Increased expression levels of gal-3 have been detected in atherosclerotic lesions (9), and the oxLDL-induced expression of gal-3 has previously been described in macrophages (24). In addition, it has been suggested that oxLDL-induced phenotypic transformation involves numerous molecular mechanisms (25). The present study provides, to the best of our knowledge, the first report that gal-3 has a critical role in the oxLDL-induced phenotypic transformation of HUSMCs. The canonical Wnt/ $\beta$-catenin signaling pathway was also reported to be associated with this process.

Abnormal proliferation of SMCs in the subendothelial space of arterial walls has an important role in the progression of atherosclerotic lesions (26). Numerous studies have demonstrated that oxLDL may promote cellular proliferation and migration $(4,21)$. A recent study reported that gal-3 acts as an anti-adhesive factor, and may promote SMC proliferation and migration, thus suggesting that gal-3 may accelerate atherogenesis $(9,27)$. The present study demonstrated that silencing of gal-3 by siRNA significantly reduced oxLDL-mediated cellular proliferation and migration in vitro. The results of the present study conclusively established that endogenous gal-3 may significantly influence oxLDL-induced proliferation and migration. Foam cell formation is an important contributor to atherosclerosis. Foam cells were previously thought to originate primarily from macrophages; however, recent studies have indicated that VSMCs are also precursors of foam cells $(28,29)$. The phenotypic transformation of VSMCs is closely associated with the phagocytosis of VSMCs, and this transformation has an important role in regulating cellular lipid accumulation (28). It has been reported that gal-3 is required for aldosterone-induced inflammation and fibrosis (6). However, the association between endogenous gal-3 and the phenotypic transformation of VSMCs remains unknown. The results of the present study demonstrated that silencing of gal-3 was able to suppress the phagocytosis of HUSMCs.

Contractile and synthetic SMCs are located on the opposite ends of the SMCs spectrum, with intermediate phenotypes 
A

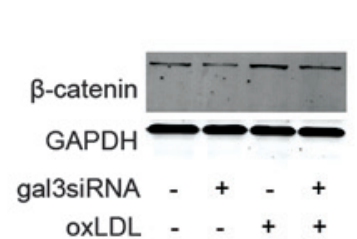

B

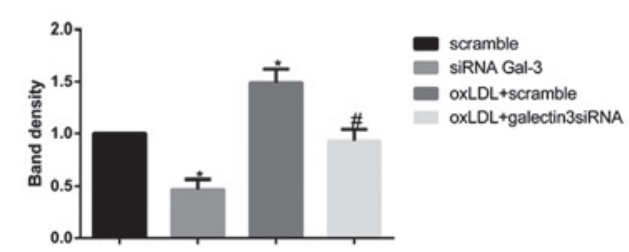

C

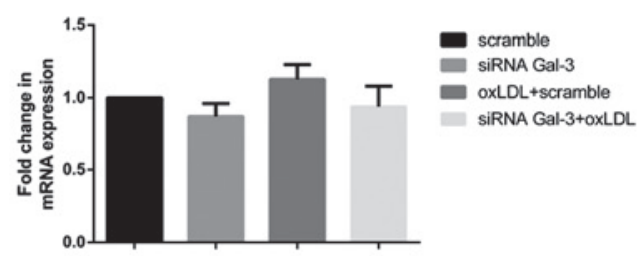

D

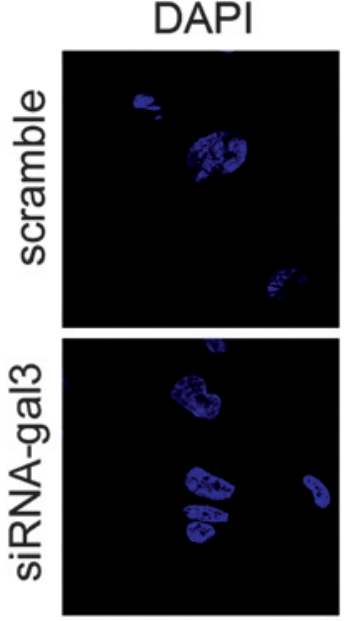

$\beta$-catenin

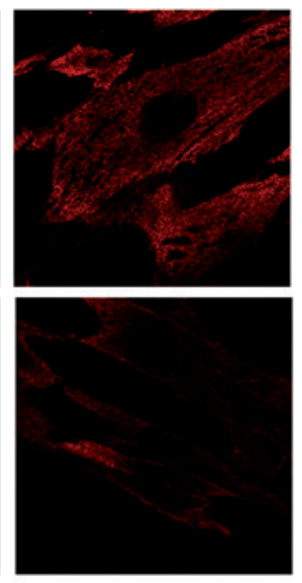

Merge

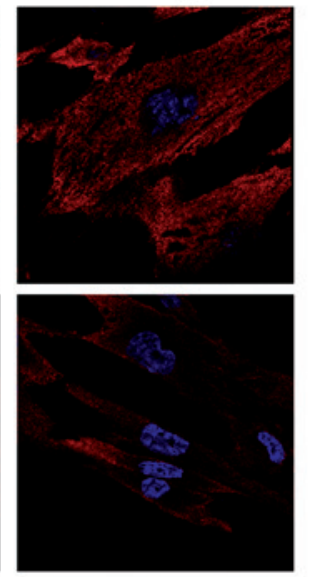

Figure 4. Galectin-3 (gal-3) silencing inhibits the expression of $\beta$-catenin. Following transfection with either control or gal-3-specific small interfering (si)RNA for $24 \mathrm{~h}$, human umbilical smooth muscle cells (HUSMCs) were incubated for $48 \mathrm{~h}$ in the presence or absence of $50 \mu \mathrm{g} / \mathrm{ml}$ oxidized low-density lipoprotein (oxLDL). $\beta$-catenin expression was measured by reverse transcription-quantitative polymerase chain reaction (RT-qPCR) and western blotting. $\beta$-catenin representative (A and B) western blotting and (C) RT-qPCR results are shown. GAPDH expression was used to normalize protein expression levels. (B) The respective densitometric measurement results are given. The band density of HUSMCs transfected with either scramble siRNA was defined as the control and set to 1 . The data are presented as the mean \pm standard deviation from three independent experiments. ${ }^{*} \mathrm{P}<0.05$, vs. the control. ${ }^{\sharp} \mathrm{P}<0.05$, vs. oxLDL. (D) The confocal images of HUSMC transfected with either control or gal-3-specific siRNA using anti- $\beta$-catenin (red) antibodies, followed by DAPI nuclear counter staining (blue) are shown. The merged images containing all markers are also shown (magnification, x630).

located in between (30). These contractile and synthetic phenotypes are regarded as "idealized" phenotypes, with heterogeneity in the phenotypes of smooth muscle cells being reported in numerous studies (30-32). In some instances, contractile differentiation may be upregulated in the synthetic phenotype (30), and contractile differentiation markers are expressed during matrix synthesis (32). Similarly, whereas higher growth rates and stronger migratory activity are usually considered typical of the synthetic phenotype, OPN has also been identified as a synthetic-associated protein (2). The results of the present study suggested that oxLDL not only increased the characteristics of the synthetic phenotype, but also promoted the activity of contractile-associated proteins calponin and SMA. Gal-3 is widely expressed in numerous types of cells, and its expression is closely correlated with the proliferation and metastasis of these various cell types $(8,33)$. Until recently, the role of gal-3 in HUSMCs was also poorly characterized. A previous study demonstrated that VSMCs were able to express gal-3 under basal conditions (6). The results of the present study correlated with these results and also suggested that the expression levels of gal-3 were moderately enhanced by oxLDL. The present study also investigated the effects of gal-3 on the process of oxLDL-induced phenotypic transformation of HUSMCs. The results suggested that gal-3 knockdown significantly inhibited both the oxLDL-induced mRNA and protein expression levels of OPN, calponin, and
SMA. Therefore, following gal-3-knockdown, oxLDL is more likely to induce the contractile phenotype.

Previous studies have reported that $\beta$-catenin has a crucial role in cell proliferation and migration $(33,34)$. Wnt/ $\beta$-catenin signaling has also been shown to be associated with OPN, SMA, and calponin expression in numerous types of cells $(23,30)$. It is well-documented that $\beta$-catenin is regulated by gal-3 in colon and gastric cancer cells $(22,35)$, and gal-3 is considered an important factor that maintains the stability of $\beta$-catenin (36). The present study therefore aimed to investigate whether $\beta$-catenin was affected by the silencing of gal-3 in HUSMCs. The results of the present study demonstrated that knockdown of gal-3 decreased the protein expression levels of $\beta$-catenin, but had no effect on its mRNA expression levels. The present study also demonstrated that oxLDL increased only the protein expression levels of $\beta$-catenin, but not the mRNA expression levels. Following cellular transfection with gal-3 siRNA, the oxLDL-induced increase in the protein expression levels of $\beta$-catenin was also inhibited. These results are concordant with those of previous studies $(36,37)$. Furthermore, binding of $\beta$-catenin to gal-3 has been shown to increase glycogen synthase kinase $3 \beta$ (GSK-3 $\beta$ ) phosphorylation. Inactivation of GSK-3 $\beta$ is able to inhibit the degradation of $\beta$-catenin, and increase the cellular levels of $\beta$-catenin (38). These results suggest that oxLDL-induced activation of the canonical Wnt/ $\beta$-catenin is 
dependent on the presence of gal-3 in HUSMCs. Canonical Wnt/ $\beta$-catenin signaling activation has been associated with cellular $\beta$-catenin distribution $(1,36)$. The present study also aimed to detect $\beta$-catenin in both the cytoplasm and the nuclei of HSMCs. The results demonstrated that $\beta$-catenin was subcellularly distributed in HUSMCs, both in the presence and absence of gal-3 siRNA transfection. In addition, silencing of gal-3 induced a decrease in the expression levels of $\beta$-catenin both in the cytoplasm and the nucleus.

In conclusion, the present study demonstrated that gal-3 is an important factor in the oxLDL-induced phenotypic transformation of VSMCs, and a mediator of oxLDL-induced activation of $\mathrm{Wnt} / \beta$-catenin signaling. However, the role of gal-3 in the phenotypic transformation of HUSMCs remains to be determined in vivo, and the mechanism by which the protein expression levels of gal-3 and $\beta$-catenin affect the function and phenotype of HUSMC merits further study. Recently, gal-3 has been identified as an important factor in atherosclerosis and heart failure $(5,39)$. The findings of the present study may provide a novel therapeutic strategy for the treatment of atherosclerosis.

\section{Acknowledgements}

The present study was supported by grants from the National Natural Science Foundation of China (grant no. 81270376); the Shanghai Hospital Development Center (grant no. SHDC12012312); the Ministry of Education Science and Technology Development Center (grant no. 20130073110016); and the Fund of the Ninth People's Hospital (grant no. 2013A02).

\section{References}

1. Mill C and George SJ: Wnt signalling in smooth muscle cells and its role in cardiovascular disorders. Cardiovasc Res 95: 233-240, 2012.

2. Rensen SS, Doevendans PA and van Eys GJ: Regulation and characteristics of vascular smooth muscle cell phenotypic diversity. Neth Heart J 15: 100-108, 2007.

3. Dumic J, Dabelic S and Flögel M: Galectin-3: An open-ended story. Biochim Biophys Acta 1760: 616-635, 2006.

4. Markowska AI, Liu FT and Panjwani N: Galectin-3 is an important mediator of VEGF- and bFGF-mediated angiogenic response. J Exp Med 207: 1981-1993, 2010.

5. Anand IS, Rector TS, Kuskowski M, Adourian A, Muntendam P and Cohn JN: Baseline and serial measurements of galectin-3 in patients with heart failure: Relationship to prognosis and effect of treatment with valsartan in the Val-HeFT. Eur J Heart Fail 15: 511-518, 2013.

6. Calvier L, Miana M, Reboul P, Cachofeiro V, Martinez-Martinez E, de Boer RA, Poirier F, Lacolley P, Zannad F, Rossignol P, and López-Andrés N: Galectin-3 mediates aldosterone-induced vascular fibrosis. Arterioscler Thromb Vasc Biol 33: 67-75, 2013.

7. Morrow DA and O'Donoghue ML: Galectin-3 in cardiovascular disease: A possible window into early myocardial fibrosis. J Am Coll Cardiol 60: 1257-1258, 2012.

8. Wesley UV, Vemuganti R, Ayvaci ER and Dempsey RJ: Galectin-3 enhances angiogenic and migratory potential of microglial cells via modulation of integrin linked kinase signaling. Brain Res 1496: 1-9, 2013.

9. Arar C, Gaudin JC, Capron L and Legrand A: Galectin-3 gene (LGALS3) expression in experimental atherosclerosis and cultured smooth muscle cells. FEBS Lett 430: 307-311, 1998.

10. Massaeli H, Hurtado C, Austria JA and Pierce GN: Oxidized low-density lipoprotein induces cytoskeletal disorganization in smooth muscle cells. Am J Physiol 277: H2017-H2025, 1999.

11. Obradovic MM, Trpkovic A, Bajic V, Soskic S, Jovanovic A, Stanimirovic J, Panic M and Isenovic ER: Interrelatedness between C-reactive protein and oxidized low-density lipoprotein. Clin Chem Lab Med 53: 29-34, 2015.
12. Steinberg D: Oxidized low density lipoprotein - an extreme example of lipoprotein heterogeneity. Isr J Med Sci 32: 469-472, 1996.

13. Auge N, Garcia V, Maupas-Schwalm F, Levade T, Salvayre R and Negre-Salvayre A: Oxidized LDL-induced smooth muscle cell proliferation involves the EGF receptor/PI-3 kinase/Akt and the sphingolipid signaling pathways. Arterioscler Thromb Vasc Biol 22: 1990-1995, 2002.

14. Leavesley DI, Schwartz MA, Rosenfeld M and Cheresh DA Integrin beta 1- and beta 3-mediated endothelial cell migration is triggered through distinct signaling mechanisms. J Cell Biol 121: 163-170, 1993.

15. Argmann CA, Sawyez CG, Li S, Nong Z, Hegele RA, Pickering JG and Huff MW: Human smooth muscle cell subpopulations differentially accumulate cholesteryl ester when exposed to native and oxidized lipoproteins. Arterioscler Thromb Vasc Biol 24: 1290-1296, 2004.

16. Livak KJ and Schmittgen TD: Analysis of relative gene expression data using real-time quantitative PCR and the 2(-Delta Delta C(T)) Method. Methods 25: 402-408, 2001.

17. Karagiannis GS, Weile J, Bader GD and Minta J: Integrative pathway dissection of molecular mechanisms of moxLDL-induced vascular smooth muscle phenotype transformation. BMC Cardiovasc Disord 13: 4, 2013.

18. Augé N, Maupas-Schwalm F, Elbaz M, Thiers JC, Waysbort A, Itohara S, Krell HW, Salvayre R and Nègre-Salvayre A: Role for matrix metalloproteinase-2 in oxidized low-density lipoprotein-induced activation of the sphingomyelin/ceramide pathway and smooth muscle cell proliferation. Circulation 110: 571-578, 2004

19. Guyton JR, Lenz ML, Mathews B, Hughes H, Karsan D, Selinger E and Smith CV: Toxicity of oxidized low density lipoproteins for vascular smooth muscle cells and partial protection by antioxidants. Atherosclerosis 118: 237-249, 1995.

20. Deng DX, Spin JM, Tsalenko A, Vailaya A, Ben-Dor A, Yakhini Z, Tsao P, Bruhn L and Quertermous T: Molecular signatures determining coronary artery and saphenous vein smooth muscle cell phenotypes: Distinct responses to stimuli. Arterioscler Thromb Vasc Biol 26: 1058-1065, 2006.

21. Ding Z, Liu S, Yang B, Fan Y and Deng X: Effect of oxidized low-density lipoprotein concentration polarization on human smooth muscle cells' proliferation, cycle, apoptosis and oxidized low-density lipoprotein uptake. J R Soc Interface 9: 1233-1240, 2012.

22. Shimura T, Takenaka Y, Tsutsumi S, Hogan V, Kikuchi A and Raz A: Galectin-3, a novel binding partner of beta-catenin. Cancer Res 64: 6363-6367, 2004.

23. Menini S, Iacobini C, Ricci C, Blasetti Fantauzzi C, Salvi L, Pesce CM, Relucenti M, Familiari G, Taurino M and Pugliese G: The galectin-3/RAGE dyad modulates vascular osteogenesis in atherosclerosis. Cardiovasc Res 100: 472-480, 2013.

24. Kim K, Mayer EP and Nachtigal M: Galectin-3 expression in macrophages is signaled by Ras/MAP kinase pathway and up-regulated by modified lipoproteins. Biochim Biophys Acta 1641: 13-23, 2003.

25. Liu J, Ren Y, Kang L and Zhang L: Oxidized low-density lipoprotein increases the proliferation and migration of human coronary artery smooth muscle cells through the upregulation of osteopontin. Int J Mol Med 33: 1341-1347, 2014.

26. Dzau VJ, Braun-Dullaeus RC and Sedding DG: Vascular proliferation and atherosclerosis: New perspectives and therapeutic strategies. Nat Med 8: 1249-1256, 2002.

27. Nachtigal M, Ghaffar A and Mayer EP: Galectin-3 gene inactivation reduces atherosclerotic lesions and adventitial inflammation in ApoE-deficient mice. Am J Pathol 172: 247-255, 2008.

28. Xue JH, Yuan Z, Wu Y, Liu Y, Zhao Y, Zhang WP, Tian YL, Liu WM, Liu Y and Kishimoto C: High glucose promotes intracellular lipid accumulation in vascular smooth muscle cells by impairing cholesterol influx and efflux balance. Cardiovasc Res 86: 141-150, 2010.

29. Rong JX, Shapiro M, Trogan E and Fisher EA: Transdifferentiation of mouse aortic smooth muscle cells to a macrophage-like state after cholesterol loading. Proc Natl Acad Sci USA 100: 13531-13536, 2003.

30. Carthy JM,Luo Z and McManus BM: WNT3A induces a contractile and secretory phenotype in cultured vascular smooth muscle cells that is associated with increased gap junction communication. Lab Invest 92: 246-255, 2012.

31. Hao H, Gabbiani G and Bochaton-Piallat ML: Arterial smooth muscle cell heterogeneity: Implications for atherosclerosis and restenosis development. Arterioscler Thromb Vasc Biol 23: 1510-1520, 2003. 
32. Rama A, Matsushita T, Charolidi N, Rothery S, Dupont E and Severs NJ: Up-regulation of connexin 43 correlates with increased synthetic activity and enhanced contractile differentiation in TGF- $\beta$-treated human aortic smooth muscle cells. Eur J Cell Biol 85: 375-386, 2006.

33. Xie D, Yin D, Tong X, O'Kelly J, Mori A, Miller C, Black K, Gui D, Said JW and Koeffler HP: Cyr61 is overexpressed in gliomas and involved in integrin-linked kinase-mediated Akt and beta-catenin-TCF/Lef signaling pathways. Cancer Res 64: 1987-1996, 2004.

34. Lee KB, Ye S, Park MH, Park BH, Lee JS and Kim SM: p63-Mediated activation of the $\beta$-catenin/c-Myc signaling pathway stimulates esophageal squamous carcinoma cell invasion and metastasis. Cancer Lett 353: 124-132, 2014.

35. KimSJ,ChoiIJ,Cheong TC,LeeSJ,LotanR,ParkSHandChunKH: Galectin-3 increases gastric cancer cell motility by up-regulating fascin-1 expression. Gastroenterology 138: 1035-1045, 2010 .
36. Kobayashi T, Shimura T, Yajima T, Kubo N, Araki K, Tsutsumi S, Suzuki H, Kuwano H and Raz A: Transient gene silencing of galectin-3 suppresses pancreatic cancer cell migration and invasion through degradation of $\beta$-catenin. Int $\mathrm{J}$ Cancer 129: 2775-2786, 2011.

37. Zhang D, Chen ZG, Liu SH, Dong ZQ, Dalin M, Bao SS, Hu YW and Wei FC: Galectin-3 gene silencing inhibits migration and invasion of human tongue cancer cells in vitro via downregulating $\beta$-catenin. Acta Pharmacol Sin 34: 176-184, 2013.

38. Song S, Mazurek N, Liu C, Sun Y, Ding QQ, Liu K, Hung MC and Bresalier RS: Galectin-3 mediates nuclear beta-catenin accumulation and Wnt signaling in human colon cancer cells by regulation of glycogen synthase kinase-3beta activity. Cancer Res 69: 1343-1349, 2009.

39. Weir RA, Petrie CJ, Murphy CA, Clements S, Steedman T, Miller AM, McInnes IB, Squire IB, Ng LL, Dargie HJ, and McMurray JJ: Galectin-3 and cardiac function in survivors of acute myocardial infarction. Circ Heart Fail 6: 492-498, 2013. 